\title{
Pulsating Stars and the Distance Scale - I
}

\author{
Alistair R. Walker \\ Cerro Tololo Inter-American Observatory ${ }^{1}$, Casilla 603, La Serena, \\ Chile
}

\begin{abstract}
A summary is given of recent measurements, by various methods, of the absolute magnitudes for RR Lyrae stars. Calibrations within our galaxy, using the Baade-Wesselink method and by Statistical Parallaxes, give a result that is some 0.2-0.3 mag fainter than is obtained by all other methods considered. The resolution of this conundrum will be likely in the near future when new and improved datasets become available.
\end{abstract}

\section{Introduction}

RR Lyrae stars are representative of the oldest recognisable stellar population. They occur in the general field, and in globular clusters. For the latter, ages are constrained by use of theoretical isochrones to fit the main-sequence turn-off, if distances are known. Since globular clusters are thought to have formed very shortly after the formation of our galaxy, their ages define a lower limit to the age of the galaxy. The distribution of field RR Lyraes in our galaxy allows us to compare population models with the observations, and to determine the size of the galaxy. In local group galaxies, the RR Lyrae distance scale can be compared with those determined from other distance indicators, such as Cepheids and Miras. The Large Magellanic Cloud (LMC) is crucial in this respect.

Recently, it has become apparent that the RR Lyrae distance scale zeropoint differs significantly depending on whether it is calibrated via the Cepheid distance scale in the LMC, or by distances determined to galactic halo RR Lyraes, using Baade-Wesselink analyses or the Statistical Parallax method. Chaboyer (1995) in a very useful discussion of all the theoretical and observational parameters affecting the ages of galactic globular clusters, points out that the uncertainty in the distances to the clusters is the single largest effect, some 20 percent. Also uncertain is the slope of the RR Lyrae absolute magnitude-metallicity relation. Some pulsation analyses (Sandage 1993, Cox 1994) indicate a high slope of $0.30-0.40$, whereas most other methods give a low slope, 0.15-0.22. Higher values imply that the galactic globular clusters are coeval, or nearly so, whereas smaller values indicate that the globular cluster system formed over a period of some Gyr.

\footnotetext{
${ }^{1}$ Cerro Tololo Inter-American Observatory, National Optical Astronomy Observatories, is operated under cooperative agreement with the National Science Foundation.
} 
The distance scale for the RR Lyraes, via the ages that result, is thus of crucial importance in the discussion of theories of formation of our galaxy, and is the best observational lower limit to the age of the Universe. In the sections that follow I will summarise the various methods recently used to determine $\mathbf{R R}$ Lyrae absolute magnitudes.

\section{Methods}

\subsection{Comparison with Cepheids in the LMC and other local group galaxies}

RR Lyraes occur in seven LMC clusters and for all of them mean magnitudes are available from CCD photometry, as summarised by Walker (1992). These clusters have a rather small range in metallicity and to a good approximation all can be assumed to have $[\mathrm{Fe} / \mathrm{H}]=-1.9$. If the $\mathrm{LMC}$ is assumed to have a distance modulus of $18.5 \mathrm{mag}$ then the RR Lyraes have $M_{v}=0.47 \pm 0.03$ The precise value depends on whether the clusters are forced to conform to some LMC geometry, such as the HI disk, but since they are well-distributed over the face of the LMC the mean absolute magnitude changes by very little. The Cepheid modulus of the LMC is often taken as $18.47 \pm 0.15$ (Feast \& Walker 1987), but more recent work (see e.g., M. Feast, this conference) would increase this value by perhaps $0.1 \mathrm{mag}$ (and with smaller error), partially due to a slightly larger modulus adopted for the Pleiades, and partially due to more recent work on the calibrating clusters (eg Turner 1993, Gieren \& Fouque 1993). This of course would make the RR Lyraes even brighter.

There have been a handful of surveys for field RR Lyraes in the MC, most recently those by Reid \& Freedman (1992) and Hazen \& Nemec (1992) deserve mention. Far more extensive is the approximately $8000 \mathrm{RR}$ Lyraes measured via the MACHO survey (K. Cook, this conference). Kinman et al. (1991) summarise the earlier observations, to find that (referred to an LMC modulus of 18.5) the field RR Lyraes have $M_{v}=0.52$. Suntzeff et al. (1992) has made a detailed comparison between RR Lyraes in the galactic halo and in the LMC, to find that the two populations appear indistinguishable when scaled by galaxian mass, and a reasonable mean metallicity for the LMC field RR Lyraes is $[\mathrm{Fe} / \mathrm{H}]=-1.6$. The result for the field RR Lyraes is consistent with that for the clusters, allowing for the expected increase in brightness at the metallicity decreases. The actual value for the slope of the magnitude-metallicity relation is not well-constrained due to the size of the errors in combination with the rather small baseline in metallicity.

An interesting test, suggested by D. Welch (private communication), is to measure field RR Lyraes in the vicinity of LMC clusters that are rich in Cepheids, thus minimising any effects due to interstellar absorption when making the Cepheid-RR Lyrae comparison. Such a test has been made for NGC 1866 (Welch \& Stetson 1993, Walker 1995). The result (for four RR Lyraes only) is consistent with the above measurements, thus the evidence from the LMC is that if the Cepheid distance scale is adopted then metal-poor RR Lyraes are relatively bright, with $M_{v} \approx 0.45$.

A convenient summary of the Cepheid-RR Lyrae comparison for other local group galaxies has been given by van den Bergh (1995). The data are much less 
extensive than for the $\mathrm{MC}$, the RR Lyraes are faint, and in some cases there are unresolved questions relating to differential reddening between the fields containing the RR Lyraes and the Cepheids. The best comparison is possibly that for IC1613, where Saha et al. (1992) find the RR Lyraes bright relative to the Cepheid measurements by Madore \& Freedman (1991), by about the same $\approx 0.3$ mag as found in the equivalent LMC comparison. For M31 and M33 the evidence is less clear, and van den Bergh (1995) shows that there appears to be better agreement for these more metal-rich galaxies (M31, M33, and our own galaxy by definition). He goes on to suggest that this might be due to the Cepheid PL relation having a metallicity-dependent zeropoint. HST measurements of Cepheids in environments of different metallicities at fixed distance (e.g., in M31 and M33), together with comparison with other distance indicators, should soon definitively be able to test this hypothesis.

\subsection{Comparison with the distance to the Galactic Centre}

It has been long realized that the RR Lyraes can be used to find the distance to the Galactic centre by looking for the maximum in space density of RR Lyraes along a line-of-sight passing close to the galactic centre. Two such analyses are those by Oort \& Plaut (1975) and Walker \& Mack (1986). Because of high interstellar absorption, the lines-of-sight possible are limited to the very few low absorption windows, such as Baade's Window, and even for these the absorption is a major problem. Working in the IR alleviates this, two recent such investigations are those of Fernley et al. (1993) in Plaut's field 3 low, and Fulbright et al. (1995), working in Baade's window. The RR Lyrae galactic centre distance can be compared with those obtained by other methods. Kerr \& Lynden-Bell (1986) adopted the simple approach of taking the mean of estimates for $R_{0}$ published between 1974 and 1986 . They found $R_{0}=8.5 \mathrm{kpc}$. Reid (1993) pursued the approach of accounting for systematic and statistical errors, and took account of covariance where necessary. He obtained $R_{0}=8.0 \pm 0.5$ kpc. Both these estimates include RR Lyraes themselves as part of the mix of distance indicators used to arrive at $R_{0}$. The analysis by Fulbright et al. (1995) for all the IR measurements (128 stars in two fields) is somewhat inconclusive, in that if the RR Lyraes are assumed to have absolute magnitudes as suggested by the LMC results above, i.e.,

$$
M_{K}=-2.33 \log P-1.18,
$$

then $R_{0}=8.8 \mathrm{kpc}$, while if a $0.3 \mathrm{mag}$ fainter zeropoint is chosen (see below) then the distance falls to $R_{0}=7.7 \mathrm{kpc}$. It thus appears that the distance to the galactic centre is not well known enough to unambiguously constrain RR Lyrae absolute magnitudes. This situation should change in the near future with new survey material, for OH/IR masers from the Leiden-Sydney survey (J. Chapman, this conference), and for Cepheids expected to be found in the 2MASS and DENIS surveys (M. Feast, this conference).

\subsection{Statistical parallax analysis}

The latest RR Lyrae statistical parallax analysis (Layden et al. 1994) uses many new proper motions ( 80 percent from the Northern PM survey) plus a homogeneous set of radial velocities and abundances by Layden (1994). There are 
now 214 stars in the sample, sufficient to allow separation into disk and halo populations. This is done by a combination of metallicity and kinematic indicators, in a number of ways. The results do not appear to be sensitive to the precise way in which the separation is made, nor on the initial assumptions needed to convert observed motions into space velocity. For the halo sample, which has mean $[\mathrm{Fe} / \mathrm{H}]=-1.6, M_{v}=0.74 \pm 0.12$, while for the disk sample with mean $[\mathrm{Fe} / \mathrm{H}]=-0.65, M_{v}=1.1 \pm 0.35$. The disk solution unfortunately has error large enough so that the slope of the magnitude-metallicity relation is not well-determined. The result for the halo stars is almost identical to previous determinations (Hawley et al. 1986, Strugnell et al. 1986) that used a smaller number of stars. A major criticism of the earlier work was that it was not possible to calculate independent solutions for the halo stars and the metal-rich stars. HIPPARCOS proper motions, available soon for at least $150 \mathrm{RR}$ Lyraes, should allow a more definitive solution for both disk and halo populations.

\subsection{Astrometric distances to globular clusters}

This is a very straightforward method which can give distances directly. In principle, all that is required is to equate dispersions of proper motions and radial velocities, after taking account of possible rotation (Cudworth \& Peterson 1988). Due to the large distances involved, a long baseline in time is needed to measure the proper motions to reasonable accuracy. Even with a baseline of several decades the errors are still large, two of the more accurate results are those for M2 by Cudworth \& Rauscher (1987), $M_{v}=0.65(+0.37,-0.31),[\mathrm{Fe} / \mathrm{H}]=-1.62$, and for M92 by Rees (1992), $M_{v}=0.44(+0.47,-0.38),[\mathrm{Fe} / \mathrm{H}]=-2.15$.

\subsection{Baade-Wesselink method}

The Baade-Wesselink method, as applied to RR Lyraes, has received extensive discussion in the recent literature (e.g., Liu \& Janes 1990a, Cacciari et al. 1992, Jones et al. 1992). All this work has used infrared K-band photometry and highquality radial velocity measurements. The phase interval considered has been restricted to that for which the atmosphere is expected to be "quiet". There are now a number of measurements for cluster RR Lyraes (e.g., Liu \& Janes 1990b, Storm et al. 1994, and references therein) as well as for the brighter field stars. No systematic differences in luminosity are found between field and cluster stars, for a given metallicity. The several groups engaged in this work obtain very consistent results, despite detail differences in the methods employed. The formal error bars are such that the magnitude-metallicity relation is defined quite accurately, typically

$$
M_{v}=0.16[\mathrm{Fe} / \mathrm{H}]+1.02 .
$$

Critics of the method note that it is difficult to estimate the systematic errors; changes to the temperature scale and to the conversion factor $(p)$ relating radial velocity to pulsation velocity could significantly alter the zeropoint of the magnitude-metallicity relation.

\subsection{Horizontal branch evolutionary models}

In the past five years several different groups have presented evolutionary tracks for stars evolving off the ZAHB (e.g., Lee \& Demarque 1990, Catelan 1993, 
Dorman 1993). They vary in their use of Los Alamos or OPAL opacities, in the helium abundance adopted, whether they use scaled solar abundances or enhance oxygen or the $\alpha$ elements, etc. However the luminosities calculated are in relatively good agreement. All predict a rather small value for the slope of the magnitude-metallicity relation. Before comparing with observations the ZAHBs should be brightened using determinations of the vertical extent of the instability strip such as those made by Sandage (1990). If this is done then a typical representation for the absolute magnitudes of $R R$ Lyraes is

$$
M_{v}=0.19[\mathrm{Fe} / \mathrm{H}]+0.81 .
$$

\subsection{Pulsation analyses}

The pulsation equation (van Albaba \& Baker 1971) can be written as

$$
\log L=1.19 \log P+0.81 \log M+4.193 \log T-13.687 .
$$

Thus, given $(P, M, T)$ luminosities can be derived. This can be done in a number of ways. The procedure used by Sandage (1993) is as follows. From plots of $\log P_{a b}$ vs. $[\mathrm{Fe} / \mathrm{H}]$ for cluster and field stars he derives the period shift at the Blue Fundamental Edge as

$$
\Delta \log P_{a b}=(-0.12 \pm 0.02)[F e / H]-(0.50 \pm 0.01) .
$$

A large value such as adopted for the coefficient of the metallicity term is supported by recent cluster analyses (e.g., Walker 1994 for M68). Masses are found from horizontal branch models, in this case those of Dorman (1993), by

$$
\log M=-0.059[\mathrm{Fe} / \mathrm{H}]-0.288 .
$$

Due to the large coefficient on the temperature term in the pulsation equation, correct representation of the temperature is critical. Using $(B-V)_{\text {mag }}$, and forcing

$$
d \log P / \operatorname{dog} T=-3.7
$$

then

$$
\log T_{e}=0.012[\mathrm{Fe} / \mathrm{H}]+3.865,
$$

and substituting in the pulsation equation,

$$
M_{b o l}(R R)_{b f e}=(0.36 \pm 0.12)[F e / H]+(1.00 \pm 0.10) .
$$

Updating using the Cox (1994) formulation of the pulsation equation, and slightly higher masses which are now to be preferred, makes very little change, and with

$$
B C=0.06[F e / H]+0.06
$$

then

$$
M_{v}(R R)=0.34[\mathrm{Fe} / \mathrm{H}]+1.00 .
$$

Thus a rather steep slope for the magnitude-metallicity equation is obtained. This has the property of agreeing with the LMC calibration for the metal poor 
stars, and also makes the age spread amongst the great majority of globular clusters small or non-existent.

A similar analysis by Fernley (1993) uses a smaller value for the period-shift coefficient $(\approx-0.07)$, and a mass relation from Kovacs et al. (1991) that implies a very small mass difference between $\mathrm{OoI}$ and OoII clusters. The pulsation equation is parameterised as a function of metallicity, and the $\mathrm{BC}$ equation originates with Kurucz (1979), and in any case is very similar to that given above. Temperatures are found via the convenient tabulation of $\mathrm{B}-\mathrm{V}$ and $\mathrm{V}-\mathrm{K}$ for temperatures, metallicities and gravities relevant to RR Lyraes derived by Lui \& Janes (1990a) from Kurucz (1979) models. Then,

$$
M_{v}=0.19[\mathrm{Fe} / \mathrm{H}]+0.84 .
$$

A third application of the pulsation equation is that by Smith \& Silbermann (1995) in their analysis of their new photometry of the M15 RR Lyrae variables. Adopting $0.75 M_{\odot}$ for the RRab stars, and using their V-R photometry with a Kurucz conversion to $T_{e}$, they find for these metal poor stars, which have $[\mathrm{Fe} / \mathrm{H}]=-2.15$, that $M_{v}=0.47 \pm 0.12$ where the error estimate accounts for only errors in their photometry and the reddening. They note that the Longmore et al. (1990) temperature scale is some $300 \mathrm{~K}$ cooler, which if adopted would make the RR lyraes have $M_{v}=0.63$.

The final method is that by Cox (1994), who avoids the temperature calibration in the following manner. Firstly, RRd masses are calculated using OPAL opacities, which results in masses of $0.70 M_{\odot}$ for OoI clusters such as M3 and M5, and $0.76 M_{\odot}$ for OoII clusters such as M15 and M68. He then draws the instability strip and mode switch boundaries from Bono \& Stellingwerf (1994) on the HR diagram, and adds the lines of constant period from the pulsation equation (updated version). The observational data (periods, relative luminosities) from the four clusters mentioned above are then fitted to these HR diagrams. The colours are used only to calculate bolometric corrections to $\mathrm{V}$, and to evaluate the reddening. He finds that the OoI clusters have $M_{v}(R R)=0.60$ and the OoII clusters $M_{v}(R R)=0.37$, thus the metal poor RR Lyraes are relatively bright and a steep slope for the magnitude-metallicity relation is implied.

\subsection{Fourier fitting}

This method involves matching RRc light curves to hydrodynamic pulsation models (Simon \& Clement 1993). If the light curves are represented by Fourier series,

$$
\text { magnitude }=A_{0}+\sum A_{j}\left(\cos j \omega t+\phi_{j}\right)
$$

and defining

$$
\begin{gathered}
R_{j 1}=A_{j} / A_{1}, \\
\phi_{j 1}=\phi_{j}-j \phi_{1}
\end{gathered}
$$

then it is found that the mass, luminosity, and temperature can be parametrised in terms of the Fourier parameter $\phi_{31}$, e.g.,

$$
\log L=1.04 \log P-0.058 \phi_{31}+2.41 \text {. }
$$


Because RRc light curves are nearly sinusoidal, light curves of excellent quality, with very well-sampled phase cover, or preferably both, are needed in order to derive accurate values of $\phi_{31}$. Only a few of the more recent CCD studies (eg Clement et al. 1992, Walker 1994, Smith \& Silbermann 1995) have produced data of the required quality. These results (for eight clusters) indicate that the RR Lyraes are relatively bright, with a small slope for the magnitude metallicity relation.

\section{Summary}

Recent work on the absolute magnitudes of RR Lyraes by a variety of methods have not yet reached convergence. Observationally, results from the HIPPARCOS mission will have a major impact, as they will provide improved statistical parallaxes for many RR Lyraes, and also provide parallaxes for subdwarfs. The latter, along with HST colour-magnitude diagrams for galactic globular clusters, will allow the measurement of relatively accurate distances to globular clusters via main-sequence fitting. The results from the present review are summarised in Table 1. In view of the domination of distance scale research by unexpected or under-estimated systematic errors, the non-inclusion of error estimates in Table 1 is deliberate.

Table 1. RR Lyrae Absolute Magnitude Estimates

\begin{tabular}{|c|c|c|c|c|c|}
\hline \multirow[t]{2}{*}{ Method } & \multicolumn{5}{|c|}{$[\mathrm{Fe} / \mathrm{H}]$} \\
\hline & -2.15 & -1.9 & -1.6 & -1.0 & -0.65 \\
\hline Stat. Parallax & & & 0.74 & & 1.1 \\
\hline Baade-Wesselink & 0.68 & 0.72 & 0.76 & 0.86 & 0.92 \\
\hline ZAHB models & 0.40 & 0.45 & 0.51 & 0.62 & 0.69 \\
\hline Puls. (Sandage) & 0.27 & 0.35 & 0.44 & 0.66 & 0.78 \\
\hline Puls. (Fernley) & 0.43 & 0.48 & 0.54 & 0.65 & 0.72 \\
\hline Puls. (Smith) & 0.47 & & & & \\
\hline Puls. (Cox) & 0.37 & & 0.60 & & \\
\hline Fourier Fits & 0.35 & 0.42 & 0.47 & 0.62 & \\
\hline Astrometry & 0.44 & & 0.65 & & \\
\hline LMC Cepheids & & 0.45 & & & \\
\hline
\end{tabular}




\section{References}

Bono, G., \& Stellingwerf, R.F. 1994, ApJS, 93, 233

Cacciari, C., Clemintini, G., Fernley, J. 1992, ApJ, 396, 219

Catelan, M. 1993, A\&AS, 98, 547

Chaboyer, B. 1995, ApJ, in press

Clement, C.M., Jakulac, M., \& Simon, N.R. 1992, ApJ, 395, 192

Cox, A.N. 1994, preprint

Cudworth, K.M., \& Peterson, R. 1988, In The Extra-galactic Distance Scale, S. van den Bergh \& C. Pritchet, ASP Conf. Ser no. 4, p. 172

Cudworth, K.M., \& Rauscher, 1987, AJ, 93, 856

Dorman, B. 1993, in The Globular Cluster - Galaxy connection, J.P. Brodie \& G.H. Smith, 11th Santa Cruz workshop

Feast, M.W., \& Walker, A.R. 1987, ARAA, 25, 345

Fernley, J. 1993, A\&A, 268, 591

Fullbright, J., Carney, B.W., Walker, A.R., \& Terndrup, D. 1995, in prep

Gieren, W.P., \& Fouqué 1993, AJ, 106, 734

Hazen, M.L., \& Nemec, J.M. 1992, AJ, 104, 111

Jones, R.V., Carney, B.W., Storm, J., \& Latham, D.W. 1992, ApJ, 386, 646

Kerr, F.J., \& Lynden-Bell, D. 1986, MNRAS, 221, 1022

Kinman, T.D., Stryker, L.L., Hesser, J.E., Graham, J.A., Walker, A.R., Hazen, M.L., \& Nemec, J.M. 1991, PASP, 103, 1279

Kovacs, G., Buchler, J.R., \& Marom, A. 1991, A\&A, 252, L27

Kurucz, R.L. 1979, ApJS, 40, 1

Layden, A.L. 1994, AJ, 108, 1016

Layden, A., Hanson, R., \& Hawley, S. 1994, Poster paper presented at the 184th meeting of the AAS

Lee, Y-W., \& Demarque, P. 1990, ApJS, 73, 709

Longmore, A.J., Dixon, R., Skillen, I., Jameson, R.F., \& Fernley, J.A. 1990, MNRAS, 247, 684

Liu, T., \& Janes, K.A. 1990a, ApJ, 354, 273

Liu, T., \& Janes, K.A. 1990b, ApJ, 360, 561

Madore, B.F., \& Freedman, W.L. 1991, PASP, 103, 933

Oort, J.H., \& Plaut, L. 1975, A\&A, 41, 71

Rees, R.F. 1992, AJ, 103, 1573

Reid, M.J. 1993, ARA\&A, 31

Reid, N., \& Freedman, W. 1994, MNRAS, 267, 821

Saha, A., Freedman, W.L., Hoessel, J.G., \& Mossman, A.E. 1992, AJ, 106, 763

Sandage, A. 1990, ApJ, 350, 603

Sandage, A. 1993, AJ, 106, 703

Simon, N.R., \& Clement, C.M. 1993, ApJ, 410, 526

Smith, H.A., \& Silbermann, N.A. 1995, AJ, in press 
Storm, J., Carney, B.W., \& Latham, D.W. 1994, A\&A, 290, 443

Suntzeff, N.B., Schommer, R.A., Olszewski, E.W., \& Walker, A.R. 1992, AJ, 104, 1743

Turner, D.G. 1993, In New Perspectives on Stellar Pulsation and Pulsating Variable Stars, J.M. Nemec \& J.M. Matthews, Cambridge University Press, p. 90

van Albaba, T.S., \& Baker, N. 1971, ApJ, 169, 311

Walker, A.R. 1992, ApJ, 390, L81

Walker, A.R. 1994, AJ, 108, 555

Walker, A.R. 1995, AJ, submitted

Walker, A.R. \& Mack, P.M. 1986, MNRAS

Welch, D.L., \& Stetson, P.B. 1993, AJ, 105, 1813

\section{Discussion}

Turner: In the distance ladder established using Cepheids, it seems that some of the steps may be unnecessary. With the recent Hubble Telescope results for Virgo Cluster galaxies, galactic cluster Cepheids can be used to establish distances beyond the Local Group without the intermediary of the LMC and Local Group galaxies. Interestingly enough, they give different distances than those based on the LMC calibrations. Any comment?

Walker: This is certainly true. However, since many of the new Cepheid distances to galaxies beyond the Local Group are referenced to the LMC, it seems likely that the LMC will continue to be used as a link in the distance scale chain.

Barnes: 1. Given the uncertainty in RR Lyrae statistical parallax this result can almost be made consistent with the Cepheid distance scale. A new HIPPARCOS based statistical parallax study of RR Lyraes (Daffyd Evans, John Fernley, Suzanne Hawley \& myself) should improve the uncertainty.

2. Also I believe there are likely enough systematic uncertainties in the BaadeWesselink analyses for RR Lyraes to give me pause in believing believing that distance scale (e.g., $v_{\text {puls }} / v_{\text {rad }}=f($ phase $)$ ).

With 1 and 2 I conclude that the faint RR Lyrae scale is less credible than the bright RR Lyrae scale and the Cepheid scale.

Laney: The B-W radii (based on JHK photometry) of Cloud and Galactic Cepheids are consistent with the usual distance scale.

Feast: With regard to your RR Lyrae distance to the centre it is interesting that Glass et al. (MNRAS in press), have found $8.7 \mathrm{kpc}$ from Miras (also based on a Cepheid distance to the LMC). 